\title{
„Solvency II in der Rechtsanwendung““ Tagung am 8. Juni 2010 in Frankfurt
}

\author{
Meinrad Dreher • Manfred Wandt
}

Online publiziert: 16. März 2011

(C) Springer-Verlag 2011

Das vorliegende Sonderheft enthält Referate und Diskussionsberichte von der rechtswissenschaftlichen Fachtagung „Solvency II in der Rechtsanwendung“, die am 8. Juni 2010 in Frankfurt stattfand. Veranstalter waren der Deutsche Verein für Versicherungswissenschaft in Kooperation mit Prof. Dr. Meinrad Dreher (GutenbergUniversität Mainz) und Prof. Dr. Manfred Wandt (Goethe-Universität Frankfurt).

Die im Januar 2010 in Kraft getretene Solvency II-Rahmenrichtlinie verändert das europäische Versicherungsaufsichtsrecht grundlegend. Neben umfassenden inhaltlichen Änderungen und Neuregelungen der Kapitalausstattung sowie der Unternehmensorganisation und -transparenz bestimmt die Solvency II-Richtlinie auch die grundsätzliche Zielsetzung der Versicherungsaufsichtsregulierung und der gesamten Versicherungsaufsicht eigenständig.

Namhafte Sachkenner des Versicherungswesens aus Politik, Aufsicht, Wirtschaft, Anwaltschaft und Wissenschaft widmeten sich auf der Tagung übergreifenden Grundsatzfragen und zentralen Kerninhalten des Solvency II-Projekts. Über Stand und Perspektiven der Solvency II-Verwirklichung referierten Prof. Dr. Karel van Hulle (EU-Kommission), Dr. Thomas Steffen (BaFin), Dr. Axel Wehling (GDV) und Prof. Dr. Manfred Wandt (Goethe-Universität Frankfurt). Prof. Dr. Meinrad Dreher (Gutenberg-Universität Mainz), Hergen Eilert (BaFin) und Dr. Peter Hemeling (Allianz SE) behandelten die Aufsicht über Aufsichtsrats- und Verwaltungsratsmitglieder

Prof. Dr. M. Dreher

Fachbereich Rechts- und Wirtschaftswissenschaften, Johannes Gutenberg-Universität Mainz,

Jakob-Welder-Weg 9, 55099, Mainz, Deutschland

e-mail: m.dreher@uni-mainz.de

Prof. Dr. M. Wandt (凶)

Institut für Versicherungsrecht, House of Finance, Goethe-Universität Frankfurt, Grüneburgplatz 1, 60323 Frankfurt, Deutschland

e-mail: wandt-lehrstuhl@jur.uni-frankfurt.de 
im System von Solvency II, die national bereits durch das „Gesetz zur Stärkung der Finanzmarkt- und der Versicherungsaufsicht" vom Juli 2009 verschärft wurde. Inhaltlich abgerundet wurde die Tagung durch Vorträge zur Aufsicht über Rückversicherungsunternehmen von Ralph Vogelgesang (Munich Re) sowie zu Insurance-linked Securities von Daniela Weber-Rey (Clifford Chance).

Die Tagung erfreute sich auf Grund der Aktualität der Themen und des Renommees der Referenten eines großen Zuspruchs. Die Veranstalter danken allen, die zum Gelingen der Tagung und zur Veröffentlichung der nachfolgenden Beiträge und Diskussionsberichte beigetragen haben.

Mainz und Frankfurt, im Dezember 2010 